\title{
A Standard Three-dimensional Imaging Protocol Must Be Established for Breast Volume Measurement
}

\author{
Chenglong Wang ${ }^{1} \cdot$ Chunjun Liu ${ }^{1}$
}

Received: 7 December 2018/ Accepted: 11 December 2018/Published online: 3 January 2019

(C) Springer Science+Business Media, LLC, part of Springer Nature and International Society of Aesthetic Plastic Surgery 2019

Level of Evidence $V$ This journal requires that authors assign a level of evidence to each article. For a full description of these Evidence-Based Medicine ratings, please refer to the Table of Contents or the online Instructions to Authors www.springer.com/00266.

We thank Hemmingsen et al. for their interest and appreciation of our work on breast volume changes during a menstrual cycle [1] and would like to respond as follows:

Three-dimensional (3D) imaging and magnetic resonance imaging (MRI) are popular techniques used for measuring breast volume. Unfortunately, neither of these techniques have proven optimal so far. Although MRI provides good resolution for breast tissue, as breast tissue is susceptible to gravity, the accuracy of MRI on breast volume is compromised [2]. Additionally, due to restrictions from the fixed shape and size of the MRI scanner coils, the breast volume tends to be affected, especially in the case of large breasts [3]. Last but not least, MRI is not considered a standard examination method in most countries due to its high cost. In the case of 3D imaging, aside from scanning in an upright position, it is quicker and more affordable than MRI. However, it is difficult to use in patients with significant ptosis. It should be noted that all aforementioned methods have their intrinsic disadvantages, what we can do is control for the systematic error and establish a standard protocol for measuring breast volume using either 3D imaging or MRI.

We agree with the comment that glandular tissue in the breast is more susceptible to hormonal changes during a

Chunjun Liu

liuchunjun@psh.pumc.edu.cn

1 Department of Aesthetic and Reconstructive Breast Surgery, Plastic Surgery Hospital, Chinese Academy of Medical Sciences and Peking Union Medical College, No. 33 Ba-DaChu Road, Shi-Jing-Shan District, Beijing 100144, China menstrual cycle. However, we support that the overall breast volume is more significant for clinical practice. For example, when looking at volume retention rate following fat grafting, surgeons are more interested in the overall changes of the breast volume and not just glandular tissue specifically.

Thank you again for your feedback on our article, we appreciate being able to discuss and share our research with experts in the field further.

\section{Compliance with Ethical Standards}

Conflict of interest The authors declare that they have no conflicts of interest to disclose.

Statement of Human and Animal Rights This article does not contain any studies with human participants or animals performed by any of the authors.

Informed Consent For this type of study, formal consent is not required.

\section{References}

1. Wang C, Luan J, Cheng $\mathrm{H}$ et al (2018) Menstrual cycle-related fluctuations in breast volume measured using three-dimensional imaging: implications for volumetric evaluation in breast augmentation. Aesthet Plast Surg 5:546. https://doi.org/10.1007/s00266018-1243-6

2. Howes BH, Watson DI, Fosh B et al (2017) Magnetic resonance imaging versus 3-dimensional laser scanning for breast volume assessment after breast reconstruction. Ann Plast Surg 78(4):455-459

3. Wang C, Luan J (2018) Magnetic resonance imaging versus 3-dimensional laser scanning for breast volume assessment after breast reconstruction. Ann Plast Surg 80(5):592

Publisher's Note Springer Nature remains neutral with regard to jurisdictional claims in published maps and institutional affiliations. 